\title{
SHOCK HEATING OF THE MERGING GALAXY CLUSTER A521
}

\author{
H. Bourdin ${ }^{1}$, P. Mazzotta ${ }^{1,2}$, M. Markevitch $^{3,4}$, S. Giacintucci ${ }^{4,5}$, and G. Brunetti ${ }^{6}$ \\ ${ }^{1}$ Dipartimento di Fisica, Università degli Studi di Roma "Tor Vergata," via della Ricerca Scientifica, \\ 1, I-00133 Roma, Italy; herve.bourdin @ roma2.infn.it \\ ${ }^{2}$ Harvard Smithsonian Centre for Astrophysics, 60 Garden Street, Cambridge, MA 02138, USA \\ ${ }^{3}$ NASA Goddard Space Flight Center, Code 662, Greenbelt, MD 20771, USA \\ ${ }^{4}$ Joint Space-Science Institute, University of Maryland, College Park, MD 20742-2421, USA \\ ${ }^{5}$ Astronomy Department, University of Maryland, College Park, MD 20742, USA \\ ${ }^{6}$ INAF-Istituto di Radioastronomia, via Gobetti 101, I-40129 Bologna, Italy \\ Received 2012 January 4; accepted 2012 December 3; published 2013 January 29
}

\begin{abstract}
A521 is an interacting galaxy cluster located at $z=0.247$, hosting a low-frequency radio halo connected to an eastern radio relic. Previous Chandra observations hinted at the presence of an X-ray brightness edge at the position of the relic, which may be a shock front. We analyze a deep observation of A521 recently performed with XMM-Newton in order to probe the cluster structure up to the outermost regions covered by the radio emission. The cluster atmosphere exhibits various brightness and temperature anisotropies. In particular, two cluster cores appear to be separated by two cold fronts. We find two shock fronts, one that was suggested by Chandra and that is propagating to the east, and another to the southwestern cluster outskirt. The two main interacting clusters appear to be separated by a shock-heated region, which exhibits a spatial correlation with the radio halo. The outer edge of the radio relic coincides spatially with a shock front, suggesting that this shock is responsible for the generation of cosmic-ray electrons in the relic. The propagation direction and Mach number of the shock front derived from the gas density jump, $M=2.4 \pm 0.2$, are consistent with expectations from the radio spectral index, under the assumption of Fermi I acceleration mechanism.
\end{abstract}

Key words: galaxies: clusters: general - galaxies: clusters: individual (A521) - galaxies: clusters: intracluster medium - shock waves

Online-only material: color figures

\section{INTRODUCTION}

Collisions between massive galaxy clusters are the most energetic events in the present universe. Part of the kinetic energy released during these collisions is dissipated through supersonic shock fronts propagating in the intracluster medium (ICM) and turbulent motions. While heating the thermal component of the ICM, shocks and turbulence may also accelerate (or reaccelerate) relativistic particles (e.g., Cassano \& Brunetti 2005; Hoeft \& Brüggen 2007; Ryu et al. 2003; Pfrommer et al. 2006; Brunetti \& Lazarian 2007; Vazza et al. 2009). Radio observations probe these complex mechanisms through the detection of diffuse synchrotron emission from the ICM, in the form of giant radio halos, Mpc-scale radio emission in the cluster central regions, and radio relics, sharp-edged radio sources in the cluster periphery (e.g., Ferrari et al. 2008; Cassano 2009; Venturi 2011; Brunetti 2011, for recent reviews).

Observable as sharp X-ray brightness and temperature discontinuities, few shock fronts have been detected so far because they can only be visible in the brightest cluster regions and in particularly favorable projections (Markevitch et al. 2002, 2005; Russell et al. 2010; Finoguenov et al. 2010; Macario et al. 2011). Peripheral radio relics are believed to be shock fronts that propagated far outside the X-ray bright region while still accelerating (or re-accelerating) electrons, which produce radio emission and quickly cool after the shock passes, resulting in a characteristic narrow feature (e.g., Ensslin et al. 1998; van Weeren et al. 2011). The physics of giant radio halos is probably more complex. Radio halos plausibly result from the (re-)acceleration and transport of relativistic particles in large turbulent regions of the ICM, although many aspects of the mechanisms generating radio-emitting electrons remain unclear (e.g., Brunetti 2011, for a recent review). Sharp radio edges (and radio relics) are frequently observed at the border of giant radio halos, suggesting a possible link between merger shocks and the generation of turbulence in the ICM (e.g., Markevitch 2010; Macario et al. 2011).

A521 is a moderately distant $(z=0.247)$ and $X$-ray luminous $\left(L_{\mathrm{X}}=(5.2+1.2) \times 10^{37} \mathrm{~W} \text {; Arnaud et al. } 2000\right)^{7}$ galaxy cluster, presenting several signatures of dynamical activity. As revealed already in ROSAT images, its X-ray and optical components appear spatially segregated, with an $\mathrm{N}-\mathrm{S}$ bimodality of the X-ray emission (Arnaud et al. 2000) and a more complex galaxy number density distribution revealing two NW/SE and NE/SW major elongations (Ferrari et al. 2003). As further shown from Chandra data analysis, the ICM in A521 exhibits an irregular thermal structure with indications for gas heating at the interface between the two main gas components (Ferrari et al. 2006). A521 exhibits a giant radio halo that is the prototype of the class of ultrasteep spectrum radio halos (Brunetti et al. 2008). Since the halo is spatially connected to a radio relic, A521 provides us with an ideal test case to investigate the effects of shocks on the properties of the thermal and non-thermal components of the ICM and their connection with giant radio halos. In this merging cluster, a shock is suggested by the presence of an $\mathrm{X}$-ray brightness edge on the SE side of the cluster coinciding with the edge of the radio relic (Giacintucci et al. 2008). The larger-scale cluster radio halo shows a very steep synchrotron spectrum supporting a picture where relativistic electrons are stochastically reaccelerated by the nonlinear interaction with turbulence in the ICM (Brunetti et al. 2008).

\footnotetext{
$70^{37} \mathrm{~W} \equiv 10^{44} \mathrm{erg} \mathrm{s}^{-1} ; \mathrm{X}$-ray luminosity in the $0.1-2.4 \mathrm{keV}$ band has been corrected for luminosity distance assuming $H_{0}=70 \mathrm{~km} \mathrm{~s}^{-1} \mathrm{Mpc}^{-1}$ and
} $\Lambda=0.7$. 
Table 1

Effective Exposure Time of Each XMM-Newton-EPIC Observation

\begin{tabular}{lccc}
\hline \hline $\begin{array}{l}\text { XMM-Newton } \\
\text { ObsIDs }\end{array}$ & Centre Coordinates & $\begin{array}{c}\text { MOS1 Effective } \\
\text { Exposure Time (ks) }\end{array}$ & $\begin{array}{c}\text { MOS2 Effective } \\
\text { Exposure Time (ks) }\end{array}$ \\
\hline O603890101 (S) & $04^{\mathrm{h}} 54^{\mathrm{m}} 22^{\mathrm{s}} .00-10^{\circ} 16^{\prime} 30 .^{\prime \prime}$ & $15.7(50.8 \%)$ & $15.7(68.9 \%)$ \\
$0603890101(\mathrm{U})$ & $04^{\mathrm{h}} 54^{\mathrm{m}} 22^{\mathrm{s}} .00-10^{\circ} 16^{\prime} 30 .^{\prime \prime}$ & $64.4(77.3 \%)$ & $64.5(74.6 \%)$ \\
\hline
\end{tabular}

Note. The fraction of the useful exposure time after solar-flare filtering is shown in brackets.

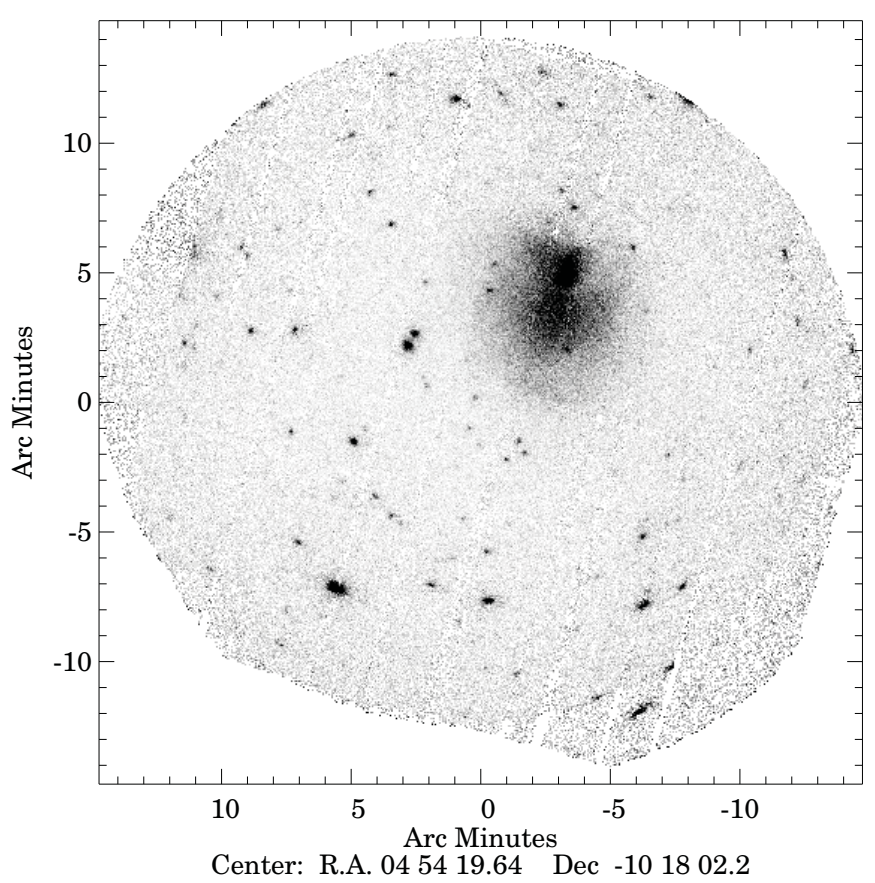

Figure 1. EPIC-XMM-Newton exposure of A521.

The present article will focus on the analysis of a deep observation of A521 recently performed with XMM-Newton, with the particular goal of probing the ICM structure up to the outermost regions covered by the cluster radio halo and radio relic. After discussing data preparation and analyses issues in Sections 2 and 3, we present the various X-ray brightness and temperature features revealed by this observation in Section 4. We comment on the interplay between thermal and non-thermal components of the ICM in Section 5. Unless otherwise noted, any energy distribution is normalized as a probability density function, while confidence ranges on individual parameter estimates are $68 \%$. In the following, intracluster distances are computed as angular diameter distances, assuming a $\Lambda \mathrm{CDM}$ cosmology with $H_{0}=70 \mathrm{~km} \mathrm{~s}^{-1} \mathrm{Mpc}^{-1}, \Omega_{\mathrm{M}}=0.3$, and $\Omega_{\Lambda}=0.7$. Given these assumptions, an angular separation of 1 arcmin corresponds to a projected intracluster distance of $232.5 \mathrm{kpc}$.

\section{OBSERVATIONS AND DATA PREPARATION}

The EPIC-XMM-Newton data set is a dual observation of A521, performed with focal aim point and central EPIC-MOS CCDs located in the SE cluster outskirts (see Figure 1). In order to remove the contribution of soft proton flares, we filtered the histogram of the photon arrival times through a temporal wavelet analysis. A summary of the "good" exposure time remaining on each of the three EPIC cameras is provided in Table 1. The average good exposure time is about $75 \mathrm{ks}$.
In order to perform imaging and spatially resolved spectroscopy, we binned photons in sky coordinates $(k, l)$ and energy $(e)$, matching the angular and spectral resolution of each focal instrument. To map the surface brightness of extended sources, these photon counts may have to be normalized for spatial and spectral variations of the telescope effective area and detector exposure times. We thus associate an "effective exposure" array, $E(k, l, e)$, with the photon event cube. Expressible, e.g., in $\mathrm{s} \mathrm{deg}{ }^{2}, E(k, l, e)$ is computed as a linear combination of CCD exposure times, $t_{\mathrm{CCD}}(k, l, p)$, related to individual observations $p$, with local corrections for useful CCD areas, $a_{\mathrm{CCD}}(k, l, p)$, Reflexion Grating Spectrometer (RGS) transmissions, ${ }^{8} t r_{\mathrm{RGS}}$, and mirror vignetting factors $a_{\mathrm{CCD}}(k, l, p)^{9}$

$$
\begin{aligned}
E(k, l, e)=\sum_{p=1}^{\mathrm{K}} t_{\mathrm{CCD}}(k, l, p) & \times \Delta a_{\text {mirror }}(k, l, e, p) \\
& \times \operatorname{tr}_{\mathrm{RGS}}(k, l, e, p) \\
& \times a_{\mathrm{CCD}}(k, l, p) .
\end{aligned}
$$

\section{DATA ANALYSIS}

\subsection{Background Noise Modeling}

The cluster emissivity must be separated from an additive, spatially extended and mostly stationary background noise, including false photon detections due to charged particleinduced and out-of-time events, but also the cosmic X-ray background (CXB), and some Galactic foreground components.

The $X M M$-EPIC background is dominated by the particle component, which is modeled from observations performed in the filter wheel closed (FWC) position during revolutions 230 to 2027 for the EPIC-MOS cameras, and 355 to 1905 for the EPIC-PN camera. Following an approach proposed in, e.g., Kuntz \& Snowden (2008) or Leccardi \& Molendi (2008), this model sums a quiescent continuum to a set of florescence emission lines convolved with the energy response of each detector. It is completed with a residual emission associated with soft protons, presently only detectable in the case of the EPIC-PN camera and modeled as a power-low spectrum normalized to $1.4 \mathrm{cts} \mathrm{deg}^{-2} \mathrm{~s}^{-1}$ in the $0.5-1$. keV band. To account for the two different spectral shapes in the soft and hard bands, the quiescent continuum is modeled as the product of a power law with an inverted error function that increases in the soft band. We set the emission line energies to the values reported in Leccardi \& Molendi (2008), while the soft proton residual is modeled using an additional power law. Presumably due to differences in the collecting areas of the imaging and readout detector regions, the EPIC-MOS quiescent continuum exhibits a small emissivity gradient along the RAWY CCD coordinate, which has been

\footnotetext{
8 EPIC-MOS detectors share a common optical path with the RGS.

9 Information about these instrumental effects has been obtained from the XMM-Newton-EPIC Current Calibration Files (CCFs).
} 
measured and taken into account in the model. Because the fluorescence lines exhibit a more complex spatial variation (Lumb et al. 2002; Kuntz \& Snowden 2008), we modeled the emissivity distribution of the most prominent lines ${ }^{10}$ from the wavelet filtering of a set of FWC event images in narrow energy intervals around each line.

Secondary background components include the CXB and Galactic foregrounds. Since they are associated with real photon detections, these components are corrected for the effective exposure. The CXB is modeled with an absorbed power law of index $\gamma=1.42$ (see, e.g., Lumb et al. 2002), while the Galactic foregrounds are modeled by the sum of two absorbed thermal components accounting for the Galactic transabsorption emission (TAE; $k T 1=0.099 \mathrm{keV}$ and $k T 2=0.248 \mathrm{keV}$, see Kuntz $\&$ Snowden 2000). We estimate the emissivities of each of these components from a "joint-fit" of all of the background components in a region of the field of view located beyond the boundary of X-ray emission in the SE cluster outskirt, but covered by the central MOS CCDs (see also Figure 2). This estimates yields 13.4, 28.0, and $29.5 \mathrm{cts} \mathrm{m}^{-2} \mathrm{deg}^{-2} \mathrm{~s}^{-1}$ in the $0.5-1$. keV band for the two transabsorption and CXB components, respectively $\left(\chi^{2} / \mathrm{dof}=1.23\right)$. Our background model, $N_{\mathrm{bck}}(k, l) \sum_{e} F_{\mathrm{bck}}$ $(k, l, e)$, eventually includes a contribution for the EPIC-PN out-of-time count rate, which is estimated in each energy band as $6.3 \%$ of all photon counts registered along the CCD columns.

\subsection{Spectroscopic and Surface Brightness Measurements}

To estimate average ICM temperatures, $k T$, and metal abundances, $Z$, along the line of sight and for a given location of the field of view $(k, l)$, we add a source emission spectrum to the background model and fit the spectral shape of the resulting function, $N_{\text {evt }}(k, l) F_{\text {evt }}\left(k T, Z, N_{\mathrm{H}}, e\right)$, to the photon energy distribution registered in the energy band $(0.3-12 \mathrm{keV})$ :

$$
\begin{aligned}
N_{\mathrm{evt}} & (k, l) F_{\mathrm{evt}}\left(k T, Z, N_{\mathrm{H}}, e\right) \\
= & E(k, l, e) \times N_{\mathrm{ICM}}(k, l) F_{\mathrm{ICM}}\left(k T, Z, N_{\mathrm{H}}, e\right) \\
& +N_{\mathrm{bck}} F_{\mathrm{bck}}(k, l, e) .
\end{aligned}
$$

In this modeling, the source emission spectrum $F_{\mathrm{ICM}}(k T, Z$, $N_{\mathrm{H}}, e$ ) assumes a redshifted and $N_{\mathrm{H}}$ absorbed emission modeled from the Astrophysical Plasma Emission Code (APEC; Smith et al. 2001), with the element abundances of Grevesse \& Sauval (1998) and the neutral hydrogen absorption cross sections of Balucinska-Church \& McCammon (1992). The $N_{\mathrm{H}}$ value has been fixed to $4.9 \times 10^{24} \mathrm{~m}^{-2}$ from measurements obtained near A521 in the Leiden/Argentine/Bonn Survey of Galactic H I (Kalberla et al. 2005). It is altered by the mirror effective areas, filter transmissions, and detector quantum efficiency (see footnote 8$)$, and convolved by a local energy response matrix $M\left(k, l, e, e^{\prime}\right)$ computed from response matrixes files (RMF) tabulated in detector coordinates in the XMM-Newton-EPIC calibration data base. ${ }^{11}$

To compute images and radial profiles of the intracluster gas distribution, we estimate a cluster surface brightness map, $\Sigma_{\mathrm{x}}(k, l)$, from photon counts registered in a given energy band and corrected for effective exposure and additive background. Assuming an average ICM energy distribution, $\left\langle F_{\mathrm{ICM}}(e)\right\rangle$, we

\footnotetext{
10 Namely, the Al, Si and $\mathrm{Cu}, \mathrm{Ni}$ complexes as for the EPIC-MOS and EPIC-PN cameras, respectively.

11 EPIC response matrixes are computed from canned RMFs corresponding to the observation period provided by the XMM-Newton Science Operation Centre.
}
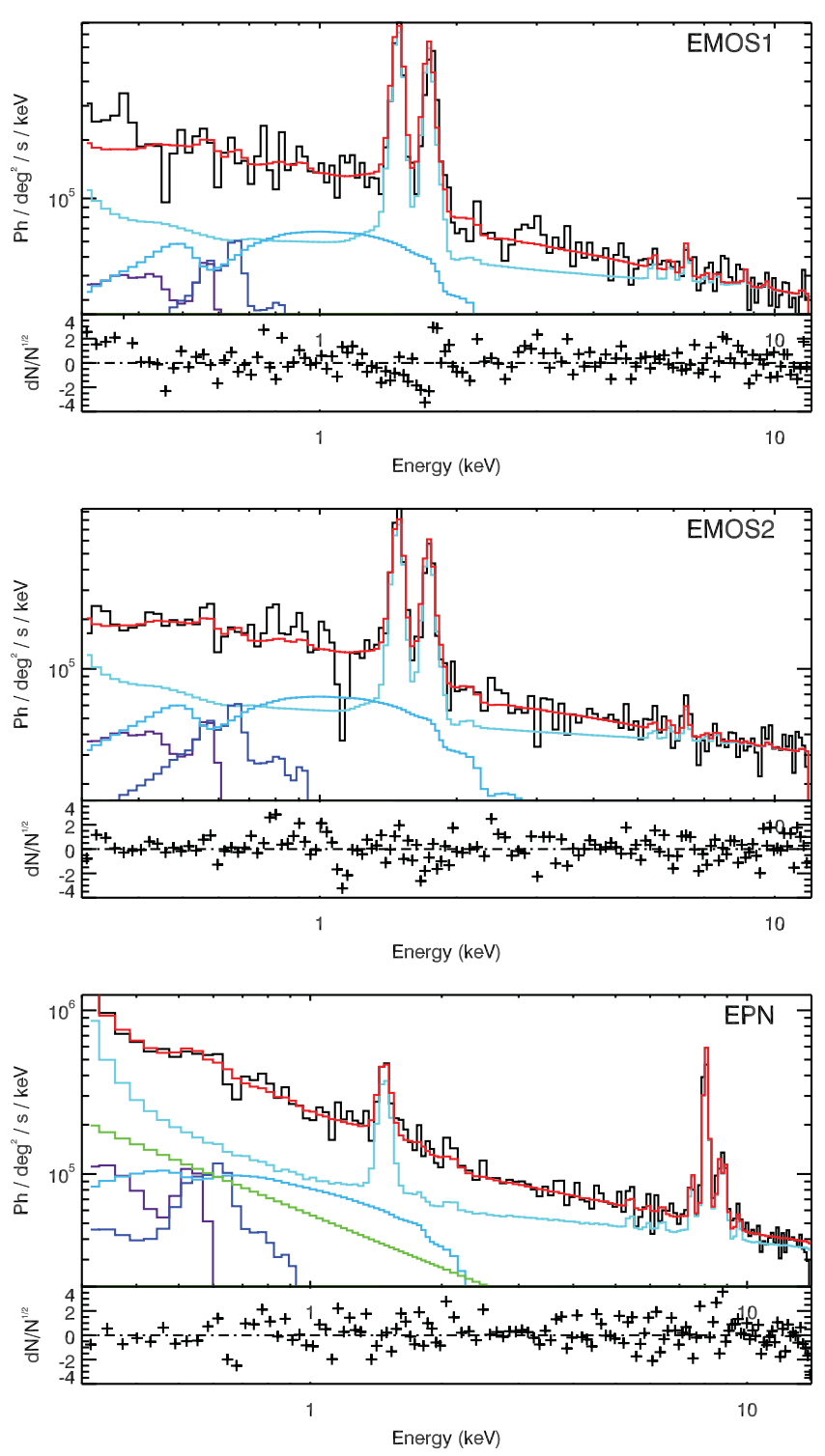

Figure 2. Background spectrum observable in the A521 outskirts. Light blue: particle background. Cyan blue: cosmic X-ray background emission. Blue and violet: TAE emission $(k T 1=0.099 \mathrm{keV}$ and $k T 2=0.248 \mathrm{keV}$, see Kuntz \& Snowden 2000, and details in Section 3.1). Green: residual soft proton emission. Red and black: overall fit and data set.

(A color version of this figure is available in the online journal.)

define $\Sigma_{\mathrm{x}}(k, l)$ as a function of an effective exposure map, $e(k, l)=\sum_{e}\left\langle F_{\mathrm{ICM}}(e)\right\rangle E(k, l, e)$ :

$$
\Sigma_{\mathrm{x}}(k, l)=\frac{\sum_{e} n_{\mathrm{evt}}(k, l, e)-N_{\mathrm{bck}}(k, l) \sum_{e} F_{\mathrm{bck}}(k, l, e)}{e(k, l)} .
$$

All parameters of $\left\langle F_{\mathrm{ICM}}(e)\right\rangle=F_{\mathrm{ICM}}\left(k T_{\mathrm{o}}, Z_{\mathrm{o}}, N_{\mathrm{H}, \mathrm{o}}, e\right)$ are practically determined from spectral fitting of the main cluster emission spectrum: $k T_{\mathrm{o}}=6.7 \mathrm{keV}, Z_{\mathrm{o}}=.4 Z_{\odot}, N_{\mathrm{H}, \mathrm{o}}=$ $4.9 \times 10^{24} \mathrm{~m}^{-2}$, while $\Sigma_{\mathrm{x}}(k, l)$ is estimated in a "soft" energy band $\left([.5-2.5] \mathrm{keV}^{\prime}\right)$ in order to lower the dependence of $\left\langle F_{\mathrm{ICM}}(e)\right\rangle$ on $k T_{\mathrm{o}}$.

\subsection{Surface Brightness, ICM Density, and Temperature Profiles}

In the following, surface brightness and temperature profiles have been extracted within cluster sectors oriented approximately along the surface brightness gradients. We derived the 
radial surface brightness $\Sigma_{\mathrm{x}}(r)$ by averaging the surface brightness $\Sigma_{\mathrm{x}}(k, l)$ of Equation (3) in each profile annulus composed of $N$ pixels $(k, l)$, as follows:

$$
\Sigma_{\mathrm{x}}(r)=\frac{1}{N} \sum_{k, l} \Sigma_{\mathrm{x}}(k, l) .
$$

Since the background contribution $N_{\mathrm{bck}}(k, l) \sum_{e} F_{\mathrm{bck}}(k, l, e)$ is estimated within a much larger area of the field of view than any sector annulus used to derive $\Sigma_{\mathrm{x}}(r)$, we neglected any systematic uncertainty related to its modeling and estimated the variance on $\Sigma_{\mathrm{x}}(r)$ from a weighted mean of the local Poisson fluctuations in $\Sigma_{\mathrm{x}}(k, l)$ :

$$
\sigma_{\Sigma_{\mathrm{x}}}(r)^{2}=\frac{1}{N} \sum_{k, l} \frac{\sigma_{n_{\mathrm{evt}}}(k, l)^{2}}{e(k, l)^{2}}=\frac{1}{N} \sum_{k, l} \frac{n_{\mathrm{evt}}(k, l)}{e(k, l)^{2}} .
$$

Projected temperatures $k T(r)$ and the associated confidence interval $\delta k T(r)$ have been computed within each annulus by fitting a uniform emission model to the data set. To do so, we averaged the emission models of Equation (2) associated with each pixel $(k, l)$ of the annulus, and estimated the model parameters $k T(r), Z(r)$, and $N_{\mathrm{H}}(r)$, via a $\chi^{2}$ minimization.

These brightness and temperature profiles have been used to model the underlying density and temperature of the ICM, assuming spherical symmetry of the cluster atmosphere in the vicinity of the features of interest. This was undertaken by projecting and fitting parametric distributions of the threedimensional (3D) emission measure ${ }^{12}, n_{p} n_{e}$, and temperature, $T(r)$, to the observed profiles. In this modeling, projected brightness profiles are convolved with the XMM-Newton focus pointspread function (PSF), while projected temperatures are computed assuming the "spectroscopic-like" weighting scheme proposed in Mazzotta et al. (2004). In Section 4.3, the ICM emission measure (see footnote 12) and temperature profiles across two shock fronts have been modeled by step-like distributions with a common jump radius $r_{j}$ :

$$
\begin{gathered}
{\left[n_{p} n_{e}\right](r)=\left\{\begin{array}{l}
D_{n}^{2} n_{o}^{2}\left(r / r_{j}\right)^{-2 \eta_{1}}, \quad r<r_{j}, \\
n_{o}^{2}\left(r / r_{j}\right)^{-2 \eta_{2},} \quad r>r_{j}
\end{array},\right.} \\
T(r)=\left\{\begin{array}{l}
D_{\mathrm{T}} T_{o}, \quad r<r_{j} \\
T_{o}, \quad r>r_{j}
\end{array}\right.
\end{gathered}
$$

\subsection{Imaging and Spectral Imaging}

\subsubsection{Imaging}

An image of the cluster is presented in the top panels of Figure 3. To obtain this image, $\Sigma_{\mathrm{x}}(k, l)$, we corrected the EPIC$X M M-N e w t o n$ raw photon image for spatially variable effective area and background flux, following Equation (3). The pointlike sources have also been modeled by means of an isotropic undecimated B3-spline wavelet analysis (see, e.g., Starck et al. 2007) and subtracted from the image.

A map of anisotropic details in the ICM structure is shown in the bottom-left panel of Figure 3. To create this image, we subtracted a wavelet filtered map of the photon rate, $\Sigma_{\mathrm{x}}(k, l)$, from the photon rate itself, then smoothed the residual image with a Gaussian function of typical width FWHM $=20$ arcsec.

\footnotetext{
12 More precisely, the ICM emission measure per volume unit.
}

The wavelet filtering was performed by means of a soft $3 \sigma$ thresholding of B3-spline wavelet coefficients, with the significance thresholds being directly computed from the raw-Poisson distributed - photon map, following the multiscale variance stabilization scheme introduced in Zhang et al. (2008).

\subsubsection{Spectral Imaging}

In order to map the ICM temperature in A521, we used the EPIC-XMM-Newton data set and applied the spectral-imaging algorithm detailed in Bourdin et al. (2004) and Bourdin \& Mazzotta (2008, hereafter B08). Following this algorithm, a set of temperature arrays $k T(k, l, a)$ with associated fluctuations $\sigma_{k t}(k, l, a)$ are first computed on various analysis scales $a$, then convolved by complementary high-pass and low-pass analysis filters in order to derive wavelet coefficients. The wavelet coefficients are subsequently thresholded according to a given confidence level in order to restore a denoised temperature map. Here, the signal analyses have been performed over six dyadic scales within an angular resolution range of $\delta a=[1.7-110]$ arcsec. This was undertaken by averaging the emission modeled by Equation (2) within overlapping meta-pixels $(k, l, a)$ and computing the $k T(k, l, a)$ and $\sigma_{k t}(k, l, a)$ arrays by means of a likelihood maximization. The resulting ICM temperature map shown in Figure 3 was then obtained from a B2-spline wavelet analysis (see B08 for details) with coefficients thresholded to the $1 \sigma$ confidence level.

\section{ICM THERMODYNAMICS}

\subsection{Intracluster Gas Brightness and Thermal Structure}

The X-ray photon image of Figure 3 reveals the complex morphology of the intracluster gas in A521. On large scales, a northern sub-cluster with a comet shape is apparently falling onto the main component. The photon image also reveals the strongly irregular morphology of the surface brightness, presenting various edges indicated with dashed lines. Some of these brightness jumps have been enhanced in the bottomleft image of anisotropic details. They are also noticeable on the surface brightness profiles of Figures 4 and 5. At the interface between the two main interacting cluster components, we observe two bow-shaped brightness jumps, $\mathrm{CF}_{1}$ and $\mathrm{CF}_{2}$, joining with each other to form a low brightness cross-shaped feature. A third brightness jump with a higher curvature radius, $\mathrm{S}_{1}$, is crossing the southern cluster component from SE to NW, while a fourth one, $S_{2}$, is visible at the southeast cluster outskirts.

The ICM temperature map of Figure 3 is strongly irregular, and presents various noticeable features. The northern subcluster is clearly cool $(k T \simeq 4.5 \mathrm{keV})$. The interacting region separating this cool core from the main cluster to the south appears hotter $(k T>7 \mathrm{keV})$ and strongly disturbed. The crossshaped brightness depression observable on the photon image seems to coincide with a hot cross $(k T \simeq 9 \mathrm{keV})$, in particular, along the brightness jump $\mathrm{CF}_{1}$. The southern part of the main cluster is cooler $(k T \simeq 4 \mathrm{keV})$ than the interacting region, in particular, to the south of the brightness jump $S_{1}$.

Bringing together the brightness and temperature maps of Figure 3, we observe that the two brightness jumps $\mathrm{CF}_{1}$ and $\mathrm{CF}_{2}$ are associated with temperature increases as the brightness decreases, while the brightness jumps $S_{1}$ and $S_{2}$ are associated with a temperature decrease. The two jumps $\mathrm{CF}_{1}$ and $\mathrm{CF}_{2}$ are thus likely to be cold fronts separating the densest parts of the two sub-clusters from their interacting region, while $S_{1}$ and 


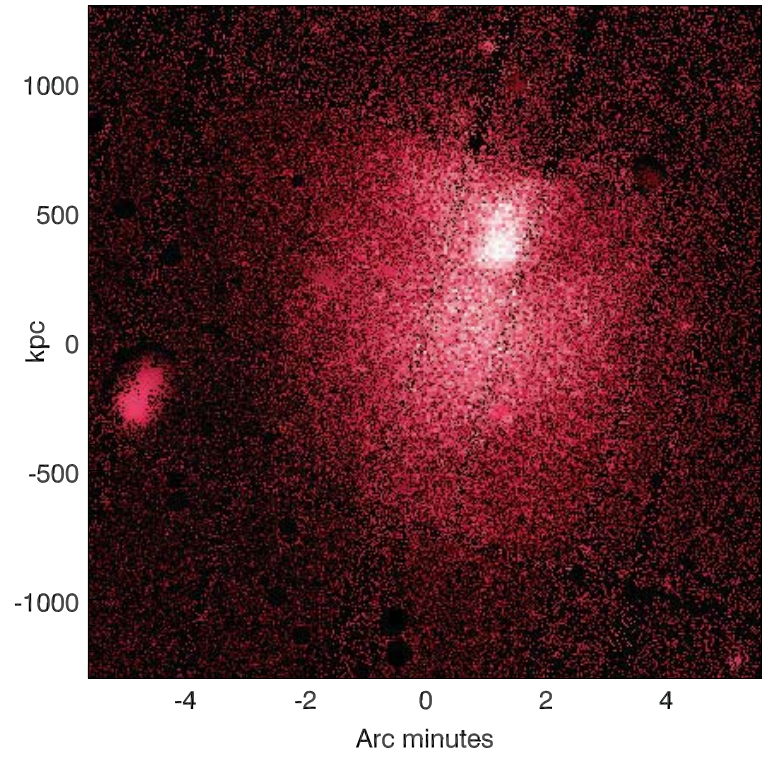

R.A.(0) $045411.40 \operatorname{Dec}(0)-101447.0$

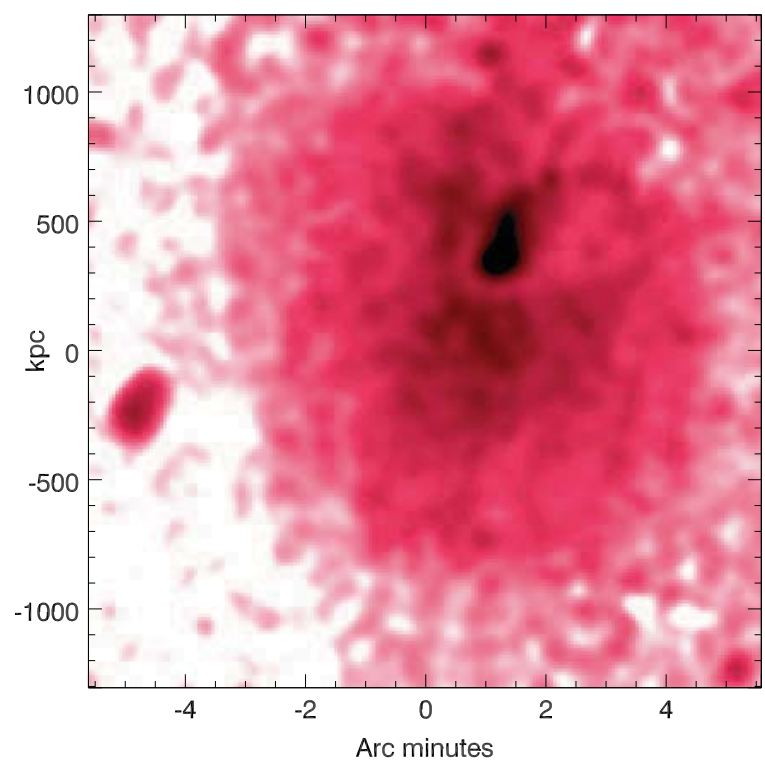

R.A.(0) $045411.40 \operatorname{Dec}(0)-101447.0$

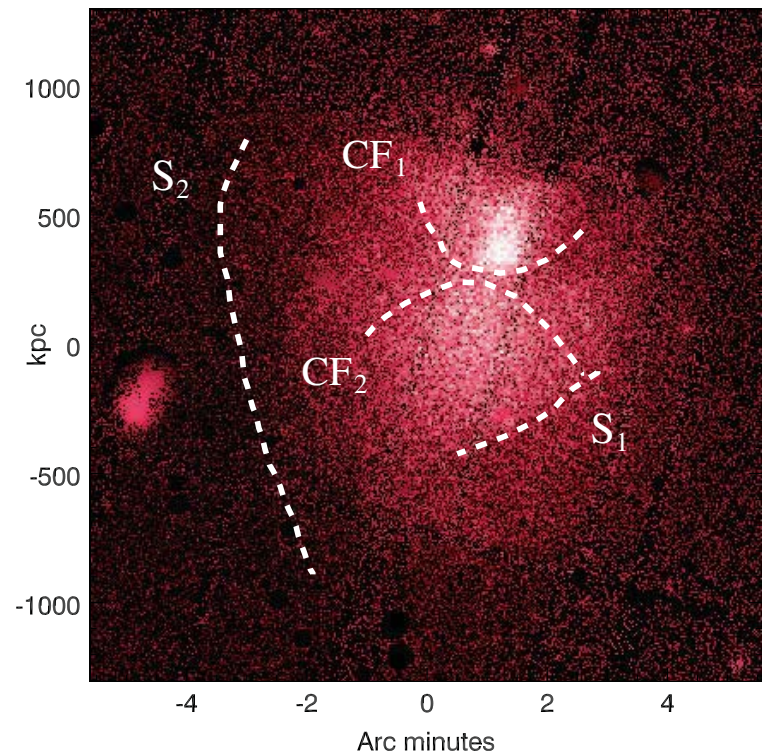

R.A.(0) $045411.40 \operatorname{Dec}(0)-101447.0$

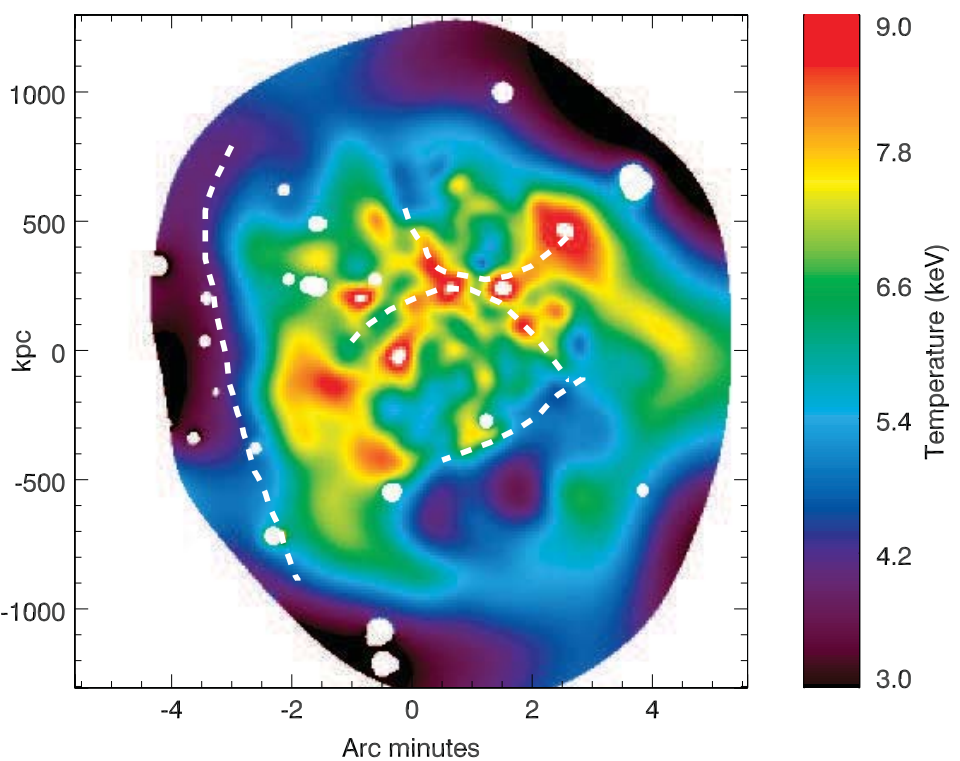

R.A.(0) $045411.40 \operatorname{Dec}(0)-101447.0$

Figure 3. EPIC-XMM-Newton observation of A521. Top panels: photon rate image in the $0.5-2.5 \mathrm{keV}$ band. Photon counts in this image have been corrected for spatially variable effective area, background flux, and wavelet-detected point-like sources. Bottom-left panel: anisotropic details in the ICM emissivity map. These details have been enhanced from subtraction of a wavelet denoised map to the photon rate (further details are provided in Section 3.4.1) Bottom-right panel: ICM temperature map obtained from wavelet spectral imaging. Prominent brightness jumps are indicated by dashed lines on the photon rate image and temperature map.

(A color version of this figure is available in the online journal.)
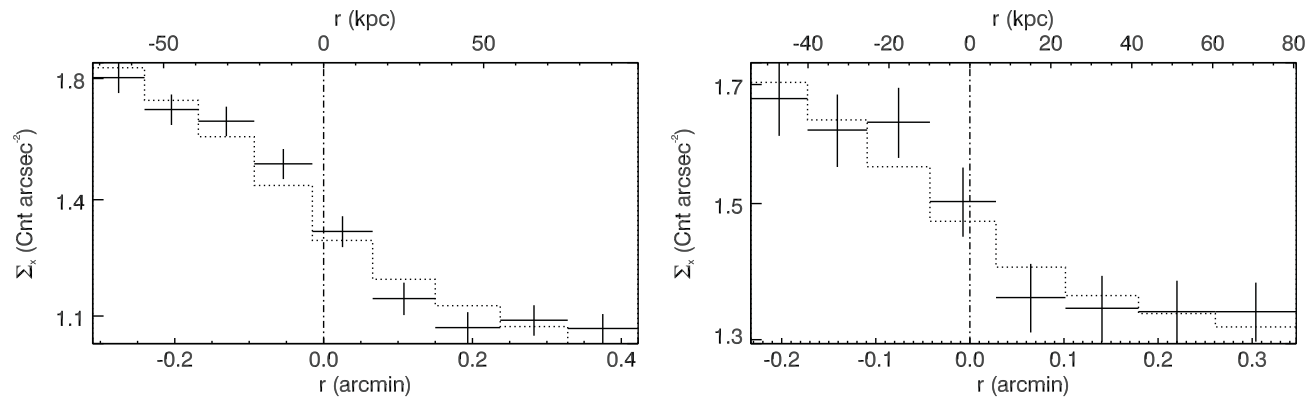

Figure 4. Projected gas brightness measured across two cluster sectors intercepting the brightness jumps $\mathrm{CF}_{1}$ and $\mathrm{CF}_{2}$. The projection of a step-like gas density distribution (Equation (6)) convolved with the XMM-Newton PSF is superimposed as a dotted line, assuming density jump amplitudes of $1.7 \pm 0.1$ and $2.1 \pm 0.1$ for $\mathrm{CF}_{1}$ and $\mathrm{CF}_{2}$, respectively. 

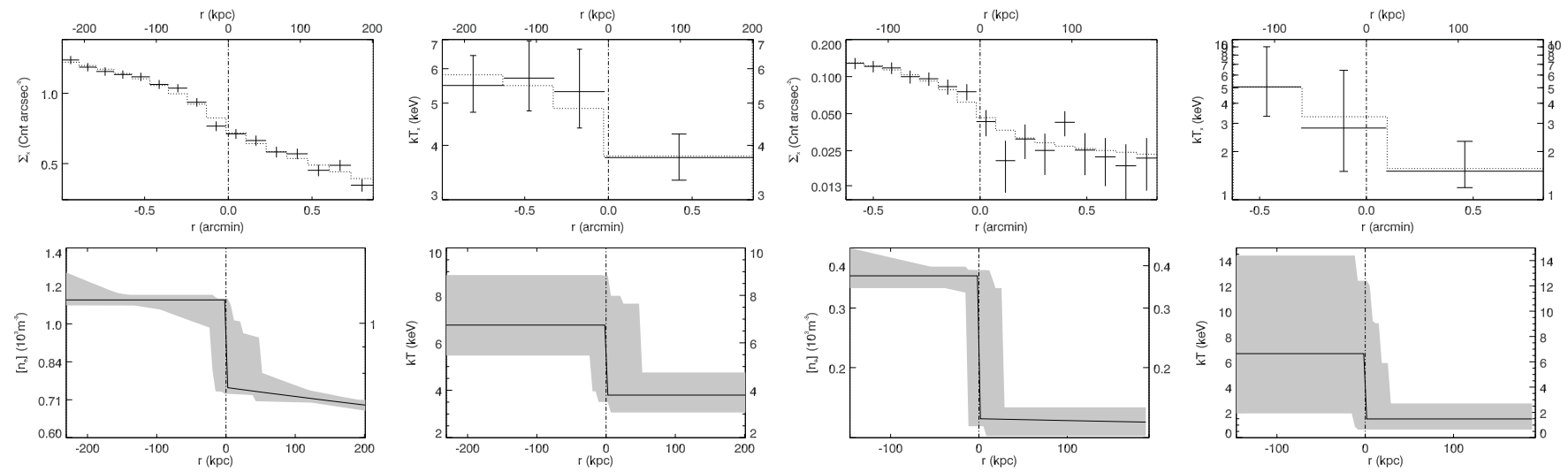

Figure 5. Top panels: projected gas brightness and temperature profiles measured across two cluster sectors intercepting the brightness jumps $S_{1}$ and $S_{2}$, as shown in Figure 7. Bottom panels: ICM density and temperature profiles modeled as step-like 3D distributions matching the projected profiles (see also Equations (6) and (7)). Dispersions on these profiles have been estimated from random realizations of the data set and corresponding models, each profile envelope delimiting $68 \%$ of the realizations with closest $\chi^{2}$ distance from the original data set. The projection of these distributions is reported as a dotted line on the projected profiles.

$\mathrm{S}_{2}$ are probably shock fronts propagating outward from the colliding clusters.

\subsection{Cold Fronts and Shock Heating at the Interface Between Two Interacting Sub-clusters}

One of the most striking features seen in our data is a cross-shaped brightness depletion separating the two colliding sub-clusters. This feature also corresponds to a temperature and entropy enhancement, in particular just outside the two cold fronts, $\mathrm{CF}_{1}$ and $\mathrm{CF}_{2}$. What we see is probably shocked gas with high entropy being squeezed by the converging cool core remnants and flowing around the densest part of the two interacting clusters without penetrating the two cold fronts. The projected layer of shocked gas would thus exhibit maximal temperature and entropy near the two cold fronts, where it is tangentially intercepted by the line of sight. This shocked gas layer might also partly overlay in projection the main subcluster from its boundary delineated by cold front $\mathrm{CF}_{2}$ to the southern brightness jump, $S_{1}$. A possible interpretation for the origin of this hot gas flow is illustrated in Figure 6. Originally located at the cluster boundary (if there is one), the high entropy gas may have been shock heated between the two clusters that were starting to interact. It would then expand over the cluster atmosphere, following the shock fronts presently propagating outside the cluster cores. One of these shock fronts might be observed to the south of the main cluster as the brightness and temperature jump, $S_{1}$.

\subsection{Shock Fronts Propagation to the Cluster Outskirts}

The 2D gas brightness and temperature maps of Figure 3 suggest that the brightness jumps $S_{1}$ and $S_{2}$ are shock fronts propagating outward from the cluster center. Located at various distances from the cluster center, these two shock fronts might have been developed during two successive cluster collisions. In order to analyze ICM thermodynamics across these jumps, we extracted the brightness and temperature profiles shown in Figure 5, corresponding to the two sectors of Figure 7. An estimate of the cluster photon counts in each sector region is provided in Table 2. The brightness jumps $S_{1}$ and $S_{2}$ exhibit the typical shape of a projected spherical density jump convolved with the XMM-Newton PSF. We model the underlying gas density and temperature profiles as two step-like functions with a common jump location, following Equations (6) and (7). A

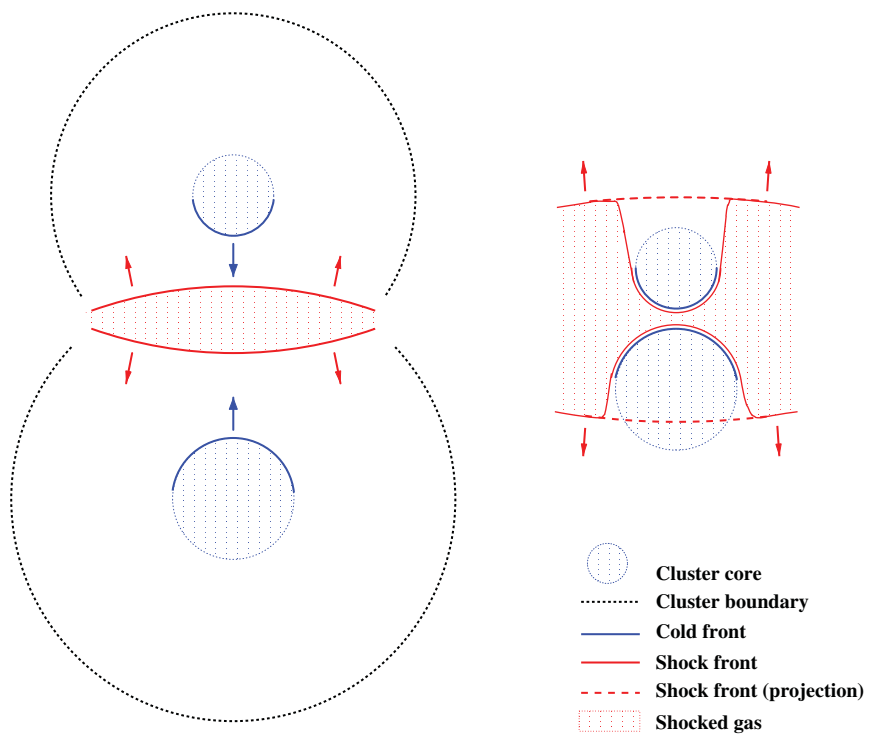

Figure 6. Tentative interpretation of the ICM thermal and entropy structure observed in the central region of A521. Left: early stage of a two-cluster merger: the cluster boundaries start to collide and develop two shock fronts propagating within the densest regions of each cluster. Meanwhile, the clusters develop two cold fronts while pushing the higher entropy gas away from their interacting region. Right: the shock fronts have now propagated to the most external regions of the interacting clusters, but could not penetrate the two cool cores. A shocked gas region with high entropy remains at the interface between the two cold fronts.

(A color version of this figure is available in the online journal.)

discussion about the validity of the assumption of the ICM spherical symmetry in the vicinity of the shock fronts is provided in the Appendix. The gas density and temperature distributions corresponding to this model are reported under the projected profiles in Figure 5. The 3D density and temperature jumps associated with these distributions are reported in Table 3, with confidence intervals estimated at $68 \%$ of a parameter sample matching several random realizations of the data set.

The direction of the temperature jumps is consistent with the shock front interpretation. The cold front hypothesis would instead imply a temperature increase across the jumps $\left(D_{\mathrm{T}}<1\right)$, which is excluded by the data. Assuming two shocks propagating outward in the main cluster, one should be able to estimate the shock Mach numbers from the Hugoniot-Rankine density, 
Table 2

Estimated Cluster Photon Counts Within the 0.3-5.0 keV Band in the Regions Shown in Figure 5

\begin{tabular}{|c|c|c|c|c|c|c|c|}
\hline \multirow[t]{2}{*}{ Detector } & \multicolumn{4}{|c|}{ Sector 1} & \multicolumn{3}{|c|}{ Sector 2} \\
\hline & Region 1 & Region 2 & Region 3 & Region 4 & Region 1 & Region 2 & Region 3 \\
\hline EPIC-MOS1 & $985(79.4 \%)$ & $518(86.1 \%)$ & $592(88.9 \%)$ & $879(90.4 \%)$ & $140(19.6 \%)$ & $173(37.0 \%)$ & $232(49.8 \%)$ \\
\hline EPIC-MOS2 & 1016(80.4\%) & $552(87.6 \%)$ & $578(89.4 \%)$ & $868(91.0 \%)$ & $87(14.9 \%)$ & $144(36.4 \%)$ & $232(49.8 \%)$ \\
\hline EPIC-PN & $2086(74.0 \%)$ & 1083(82.3\%) & $1146(86.4 \%)$ & $1557(87.3 \%)$ & $192(13.6 \%)$ & $284(29.2 \%)$ & $420(42.9 \%)$ \\
\hline
\end{tabular}

Note. The fraction of the total counts is shown in brackets.

Table 3

Density, Temperature Jumps, and Mach Numbers Estimated Across the Shock Fronts $\mathrm{S}_{1}$ and $\mathrm{S}_{2}$

\begin{tabular}{lccc}
\hline \hline Shock Front & \multicolumn{2}{c}{ Density Estimates } & \multicolumn{2}{c}{ Temperature Estimates } \\
\cline { 2 - 3 } & Jump Amplitude & Mach Number & Jump Amplitude \\
\hline $\mathrm{S}_{1}$ & $1.48_{-0.11}^{+0.02}$ & $1.33_{-0.08}^{+0.02}$ & $1.78_{-0.38}^{+0.63}$ \\
$\mathrm{~S}_{2}$ & $2.64_{-0.15}^{+0.13}$ & $2.42_{-0.19}^{+0.19}$ & $4.47_{-3.00}^{+8.06}$ \\
\hline
\end{tabular}

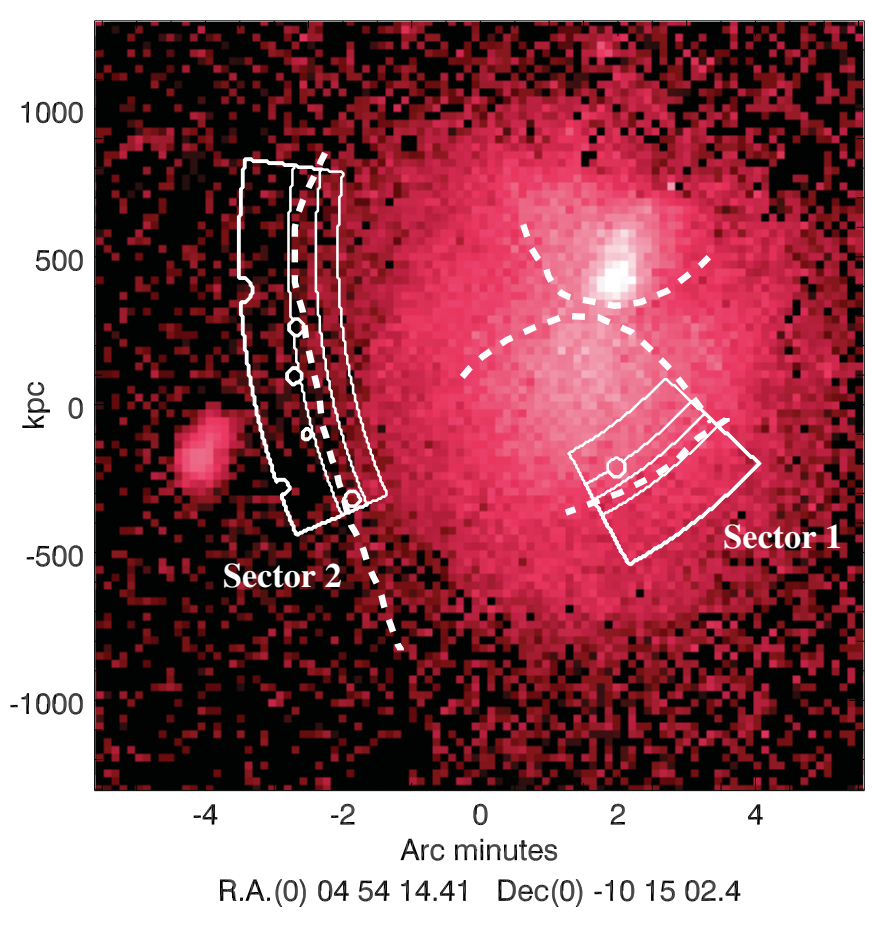

Figure 7. Photon rate image of A521 extracted in the $0.5-2.5 \mathrm{keV}$ band. The image has been re-binned to a 6.8 arcsec angular resolution in order to enhance the brightness jumps $S_{1}$ and $S_{2}$. The two annular sectors show the two cluster regions where the temperature and brightness profiles of Figure 5 have been extracted.

(A color version of this figure is available in the online journal.)

temperature, or pressure jump conditions across the fronts. Such Mach number values are reported in Table 3. The Mach numbers independently estimated from the density and temperature jumps are consistent with each other, though estimates from the temperature jumps have larger uncertainties. We will hereafter use Mach number estimates for both shocks $\mathrm{S}_{1}$ and $\mathrm{S}_{2}$, from their density jumps: $M_{\mathrm{S} 1, \rho}=1.33_{-0.08}^{+0.02}$ and $M_{\mathrm{S} 2, \rho}=2.42 \pm 0.19$.

\section{NON-THERMAL ICM EMISSION}

A521 hosts a radio relic in its southeastern peripheral region and a rare low-frequency giant radio halo. In order to investigate the interplay between thermal and non-thermal components of the ICM emission, the $240 \mathrm{MHz}$ radio image obtained from observations performed at the Giant Metrewave Radio Telescope has been superimposed on the X-ray photon and ICM temperature maps of Figure 8.

\subsection{The A521 Radio Halo}

The A521 radio halo was discovered from low-frequency observations at the GMRT $(240,325,610 \mathrm{MHz}$; Brunetti et al. 2008, see also Figure 8) and then studied in detail through a deep follow-up Very Large Array observation at $1.4 \mathrm{GHz}$ (Dallacasa et al. 2009). Its very steep spectrum, with spectral index $\alpha \sim 1.9$ between 325 and $1400 \mathrm{MHz}$, suggests magnetohydrodynamic turbulence to be responsible for the in situ re-acceleration of the relativistic electrons (Brunetti et al. 2008). The radio halo covers the cluster central region, exhibiting an EW elongation and reaching the radio relic to the southeast. When excluding the relic region, the halo appears to be spatially correlated with the cluster X-ray emission. There is an even better correlation between the radio brightness and the hottest regions of the ICM - in particular, the radio brightness exhibits a quick drop across the $S_{1}$ shock.

The complex thermodynamics of the ICM in the cluster center hint at the possible origin of the turbulence that may re-accelerate non-thermal particles in the halo. The two cold fronts $\mathrm{CF}_{1}$ and $\mathrm{CF}_{2}$ may have developed $\mathrm{K}-\mathrm{H}$ instabilities at large angles from the main cluster collision axis. As suggested by the spatial correlation between shock-heated regions and the radio emission, turbulence may alternatively have been generated behind the two shocks $S_{1}$ and $S_{2}$, now propagating to the cluster outskirts. In addition, the merger disturbance has likely generated turbulence within the two sub-cluster core remnants.

\subsection{Shock Front Propagation and the Radio Relic}

A521 has been known to host an SE radio relic observed at various frequencies (Ferrari et al. 2006; Giacintucci et al. 2006, 2008, hereafter, G08). As shown in G08, the integrated synchrotron radiation in the relic exhibits a power-law spectrum with spectral index $\alpha \simeq 1.5$ in the frequency range 235-4890 Mhz, with evidence of a steepening of the radio spectrum with increasing distance from the eastern edge. As further noted in G08, the outer edge of the radio relic coincides with the X-ray edge $S_{2}$, which we have shown in this work to be a shock 

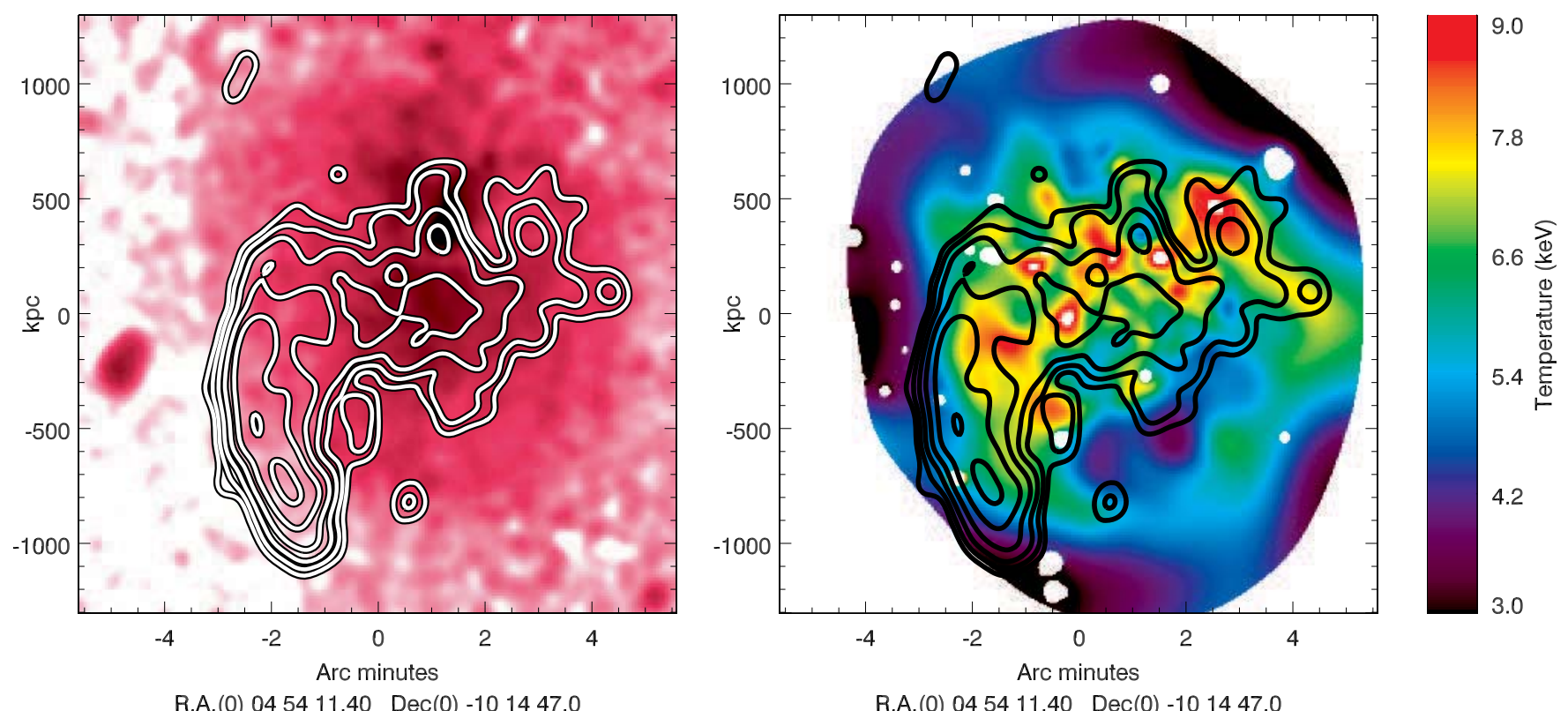

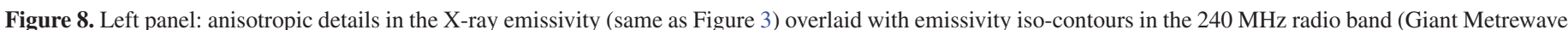
Radio Telescope; Brunetti et al. 2008). Right panel: ICM temperature map overlaid with the same radio emissivity iso-contours as for the left figure.

(A color version of this figure is available in the online journal.)

front propagating towards the cluster outskirts. As observed in several peripherical radio sources of galaxy clusters (see, e.g., Brüggen et al. 2012 for a recent review), these facts support the shock electron (re-)acceleration being at least partly responsible for the radio emission from the relic. Assuming diffuse shock acceleration for the origin of the emitting electrons, in the test particle approach, the slope of the injection spectrum of cosmic rays is related to the shock Mach number, $M$, by (Blandford \& Eichler 1987) $\delta_{i}=2\left(M^{2}+1\right) /\left(M^{2}-1\right)$. This leads to a spectrum of electrons in the downstream region with slope $\delta=\delta_{i}+1$ (implying a synchrotron spectral index $\alpha=\delta_{i} / 2$ ), taking into account radiative losses and assuming stationary conditions. G08 thus predicted a shock propagation with Mach number $M \simeq 2.3$ from their measurement of $\alpha$. The steepening of the radio spectrum with increasing distance from the eastern edge further allowed them to predict a shock propagation to the cluster outskirts. The propagation direction and Mach number of the shock front $\mathrm{S}_{2}, M_{\rho}=2.4 \pm 0.2$ (see Section 4.3), are fully consistent with this hypothesis.

Furthermore, note that the X-ray edge corresponding to the shock front seems to extend in the north-south direction more than the radio relic. A first interpretation for this limited extent of the relic might be that the shock would re-accelerate preexisting relativistic electrons in the ICM. In this case, the radio relic could reflect the spatial and energy distribution of the pre-existing electrons across the shock front. In line with this hypothesis, recent analyses (Kang \& Jones 2007; Kang \& Ryu 2011a, 2011b) suggest that the presence of pre-existing particles, in addition to the thermal pool, can significantly increase the average efficiency of the particle acceleration and the expected synchrotron emission at weak shocks $(M \leqslant 3)$. Differences in extension between the shock and the radio relic might alternatively indicate some changes in the efficiency of electron acceleration changes along the shock front, possibly due to local variations of the Mach number (e.g., Hoeft et al. 2008). In this respect, the radio relic in A521 is located at the extremity

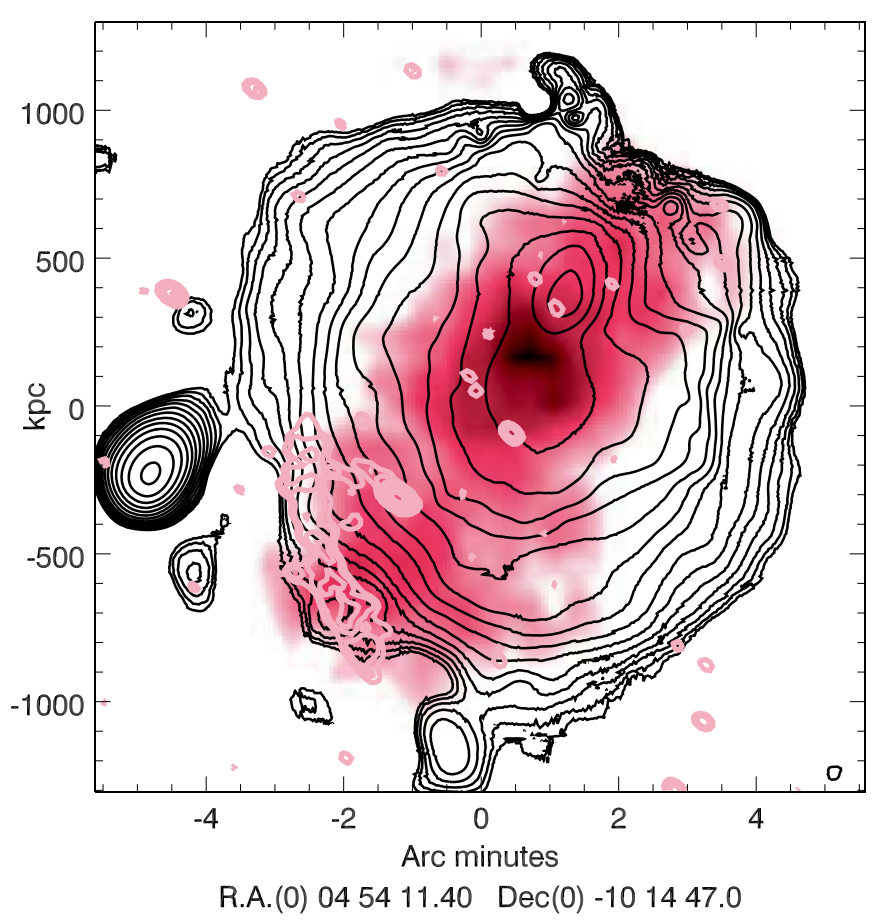

Figure 9. Projected galaxy density distribution derived from photometric observations performed at the CFH telescope (dark color indicate higher densities, Dressler algorithm; see Ferrari et al. 2003). Pink iso-contours: radio emissivity in the $610 \mathrm{MHz}$ band (Giacintucci et al. 2008). Black iso-contours: $\mathrm{X}$-ray emissivity in the $0.5-2.5 \mathrm{keV}$ band (curvelet denoising of the XMM-Newton image).

(A color version of this figure is available in the online journal.)

of the NW/SE major galaxy alignment evidenced in Ferrari et al. (2003, see also Figure 9), where, indeed, recent accretion of sub-cluster material may have produced inhomogeneities in the ICM. 


\section{DISCUSSION AND CONCLUSIONS}

A521 is a complex cluster system where optical analyses have revealed at least three galaxy groups to the SE and four groups to the NW, including the cluster BCG group coinciding with the X-ray peak (Ferrari et al. 2003). The X-ray morphology of the BCG group suggests an infall along an NNW-SSE direction (projected onto the sky plane), which is slightly offset with respects to the major NW-SE galaxy alignment (Ferrari et al. 2006). The cluster atmosphere exhibits various brightness and temperature edges associated with cold fronts and shock fronts, which were revealed by our XMM-Newton data.

The two main interacting gas components in the central region of this system are separated by a region of gas with lower density, higher temperature, and entropy. We interpret this feature as a flow of high-entropy gas being squeezed by two converging sub-cluster cores that are delimited by cold fronts. We suggest that this high entropy gas was heated by shocks formerly developed when the two gas components started to interact. One of these shocks is currently observed to the south of the main component, with Mach number $M_{\rho}=1.33_{-0.02}^{+0.02}$. The hot gas region separating the two interacting components appears spatially correlated with the cluster radio halo. The development of turbulence in the hot gas flowing between the two cool cores may be responsible for high-energy electron reacceleration, yielding the radio halo emission. Merger shock propagation and/or cold fronts may have contributed to the development of these instabilities.

A shock front is observed at the southeast cluster outskirt. An X-ray brightness edge there was hinted at by Chandra data (G08), though the statistical significance was marginal. The orientation of this shock front and its large distance from the cluster center suggest that it is associated with a cluster collision that has occurred prior to the current two-component interaction. Our Mach number for this shock, $M_{\rho}=2.4 \pm 0.2$, is consistent with that expected from the spectrum of the radio relic in G08 under the assumption of the Fermi I acceleration mechanism. As observed in X-ray follow-ups of other radio relics-A3667 (Finoguenov et al. 2010) and RXCJ1314.4-2515 (Mazzotta et al. 2011) - its detection supports the shock electron (re)acceleration to be at least partly responsible for the radio emission from the relic. The detection of a polarization of the relic would be an additional support for this process, complementary to the extension of its synchrotron spectrum to very high radio frequencies and to evidence for spectral steepening downstream of the shock (G08). Delimited by the shock front, the radio relic seems, however, to subtend only a fraction of the shock front. Differences in the spatial extent of a radio relic and its companion shock front have also been observed in the colliding cluster RXCJ1314.4-2515 (Mazzotta et al. 2011), where a radio relic seems to be confined to a small section of the shock front presumably distorted by a nonuniform gas flow. These differences may thus reflect variations of the efficiency of particle acceleration across the shock that could be driven by local variations of the Mach number and shock velocity. Deeper X-ray or SZ observations may enable us to investigate this hypothesis, though the present XMM-Newton image does not seem to provide evidence of any strong variation in the amplitude of the surface brightness edge, and thus in the shock Mach number. An alternative hypothesis is that the radio relic would reveal local inhomogeneities in the properties of the pre-existing relativistic electrons that would be re-accelerated by the shock passage. The observed connection between the radio halo and the relic may suggests that pre-existing relativistic electrons have first been accelerated by turbulent gas motions responsible for the radio halo emission, then re-accelerated at the shock front.

We thank the reviewer for constructive comments and suggestions aimed at improving the manuscript. H.B. thanks the Harvard-Smithsonian Centre for Astrophysics, where this work was initiated, for its hospitality. We thank Chiara Ferrari for providing us with a map of the projected galaxy density distribution in A521, derived from photometric observations performed at the $\mathrm{CFH}$ telescope. This work is based on observations obtained with XMM-Newton, an ESA science mission funded by ESA Member States and the USA (NASA). H.B and P.M. acknowledge support by grants NASA grant NNX09AP45G and NNX09AP36G grant ASI-INAF I/088/06/0 and ASI-INAF I/009/10/0. S.G. acknowledges the support of NASA through Einstein Postdoctoral Fellowship PF0-110071 awarded by the Chandra X-ray Center, which is operated by the Smithsonian Astrophysical Observatory. G.B. acknowledges partial support from PRIN-INAF2009.

\section{APPENDIX \\ APPENDIX MATERIAL}

The ICM density and temperature distributions intercepting the shocks $S_{1}$ and $S_{2}$ have been modeled in Section 3.3 as two step-like functions, assuming the shock center of curvature and the ICM centroid to coincide with each other.

The X-ray image of Figure 3 seems, however, to show that the shock fronts $\mathrm{S}_{1}$ and $\mathrm{S} 2$ are less curved than the closest cluster brightness isophotes. To investigate the systematic uncertainties inherent to our spherical symmetry approximation, we alternatively tried to model the shock front and the ICM density as two spherical distributions with distinct centers. Assuming these two centers to be located in the plane of the sky, the ICM emission measure is now expressed per volume unit, as

$$
\left[n_{p} n_{e}\right](r)=\left\{\begin{array}{cc}
D_{n}^{2} n_{o}^{2}\left(\frac{r^{\prime}}{r_{o}}\right)^{-\eta}, \quad r<r_{j} \\
n_{o}^{2}\left(\frac{r^{\prime}}{r_{o}}\right)^{-\eta}, \quad r>r_{j}
\end{array}\right.
$$

where $r$ and $r^{\prime}$ refer to the norm of each radius vector in the shock and ICM frames, respectively. Introducing $d$ and $d^{\prime}$, the projection of these radius vectors onto the sky plane, a surface brightness profile intercepting the shock is obtained by the integration of Equation (A1) along the line of sight:

$$
\begin{aligned}
\Sigma(x)= & 2 \times \int_{\phi_{\min }}^{\phi_{\max }} \int_{0}^{\infty} \int_{x-\delta x}^{x+\delta x} \Lambda(T(d, l))\left[n_{p} n_{e}\right] \\
& \times\left(d, d^{\prime}, l, \phi\right) d d d l d \phi,
\end{aligned}
$$

where $d^{\prime}$ is related to $d$ as a function of $r_{o}$, the distance separating the shock from the center of the ICM distribution and $\phi$, the angle separating the projected radius vector to the shock propagation axis $\left(d^{\prime}=\sqrt{\left(r_{j}-r_{o}\right)^{2}-2\left(r_{j}-r_{o}\right) d \cos (\phi)+d^{2}}\right.$, see also Figure 10). In addition to an ICM density slope, $v$, and the shock curvature radius, and density and temperature jumps, $r_{j}, D_{n}$, and $D_{T}$, respectively, the ICM emission measure thus depends on an asphericity parameter: $1-r_{o} / r_{j}$.

We tried to invert $\left[n_{p} n_{e}\right](r)$ and its parameters from a minimization of the $\chi^{2}$ distance separating $\Sigma(x)$ (Equation (A2)) 

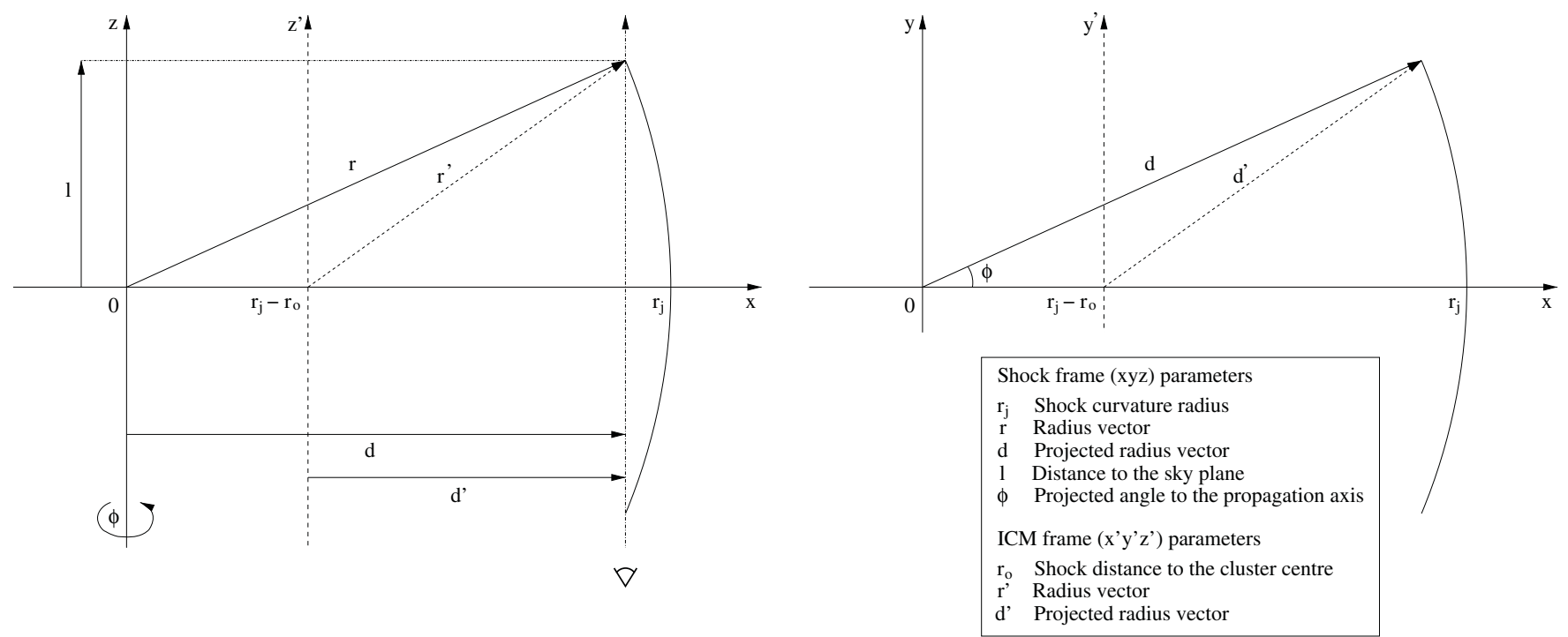

Figure 10. ICM geometry across the shocks. Left: cluster volume cut along the line of sight. Right: cluster volume cut within the sky plane.

Table 4

ICM Asphericity Parameters Across the Shock Fronts S1 and S2

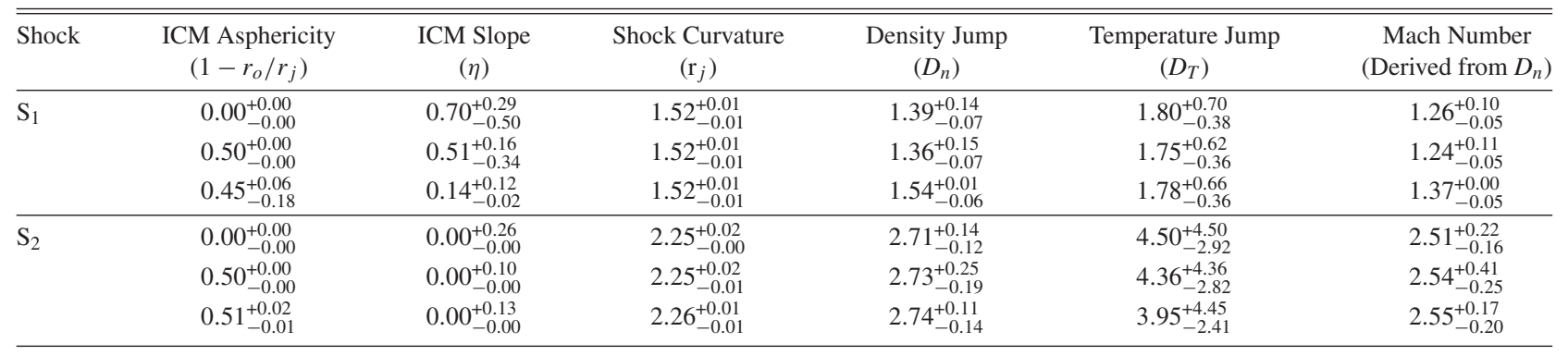

from the X-ray surface brightness profiles extracted across each shock front (see Figure 5). Since some of the searched parameters degenerate with one another, we first performed this inversion by fixing the asphericity parameter to 0 and 0.5 , corresponding to shocks located at distances of $r_{j}$ and $2 \times r_{j}$ from the cluster center, respectively. We subsequently left all parameters free to vary and report the results of our measurements in Table 4, with the confidence interval on each parameter being estimated from the $68 \%$ of a parameter sample matching several random realizations of the data set. As expected, the shock curvature radius, density, and temperature jumps obtained when fixing the asphericity to zero are consistent with their estimates derived from the spherical model of Section 3.3. A marginal difference in the amplitude of the density jump is still noticeable, since Equation (A1) yields $D_{n}=1.39_{-0.07}^{+0.14}$ for shock $\mathrm{S}_{1}$, while Equation (6) yields $D_{n}=1.33_{-0.08}^{+0.02}$. This difference is probably related to the lack of any variation of the ICM density slope across the shock, following Equation (A1). Fixing the asphericity to 0.5 instead of 0 also marginally affects the density jump, essentially due to the degeneracy between the ICM asphericity and density slope. This degeneracy is noticeable in the case of $S_{1}$, the shock front observed with the highest statistics. Leaving the ICM asphericity free to vary yields estimates of 0.45 and 0.75 in the cases of $S_{1}$ and $S_{2}$, respectively, consistent with the shock curvature radii observed on the X-ray image of Figure 3. The shock density, temperature jump, and Mach numbers derived from these various assumptions are, in any case, consistent with one another, and with their estimates obtained from the spherical model of Section 3.3. Given the limited statis- tics available, it is difficult to break the degeneracy between the ICM asphericity, ICM density slope, and shock curvature radius in the vicinity of the shocks. For simplicity, we consequently adopted the spherical model of Section 3.3 in order to derive the amplitudes of the density jumps and Mach numbers of the two shocks $S_{1}$ and $S_{2}$.

\section{REFERENCES}

Arnaud, M., Maurogordato, S., Slezak, E., \& Rho, J. 2000, A\&A, 355, 461 Balucinska-Church, M., \& McCammon, D. 1992, ApJ, 400, 699

Blandford, R., \& Eichler, D. 1987, PhR, 154, 1

Bourdin, H., Sauvageot, J., Slezak, E., Bijaoui, A., \& Teyssier, R. 2004, A\&A, 414, 429

Bourdin, H., \& Mazzotta, P. 2008, A\&A, 479, 307

Brüggen, M., Bykov, A., Ryu, D., \& Röttgering, H. 2012, SSRv, 166, 187

Brunetti, G. 2011, MmSAI, 82, 515

Brunetti, G., Giacintucci, S., Cassano, R., et al. 2008, Natur, 455, 944

Brunetti, G., \& Lazarian, A. 2007, MNRAS, 378, 245

Cassano, R. 2009, in ASP Conf. Ser. 407, The Low-Frequency Radio Universe, ed. D. J. Saikia, D. A. Green, Y. Gupta, \& T. Venturi (San Francisco, CA: ASP), 223

Cassano, R., \& Brunetti, G. 2005, MNRAS, 357, 1313

Dallacasa, D., Brunetti, G., Giacintucci, S., et al. 2009, ApJ, 699, 1288

Ensslin, T. A., Biermann, P. L., Klein, U., \& Kohle, S. 1998, A\&A, 332, 395

Ferrari, C., Arnaud, M., Ettori, S., Maurogordato, S., \& Rho, J. 2006, A\&A, 446, 417

Ferrari, C., Govoni, F., Schindler, S., Bykov, A. M., \& Rephaeli, Y. 2008, SSRv, 134, 93

Ferrari, C., Maurogordato, S., Cappi, A., \& Benoist, C. 2003, A\&A, 399, 813 Finoguenov, A., Sarazin, C. L., Nakazawa, K., Wik, D. R., \& Clarke, T. E. 2010, ApJ, 715, 1143

Giacintucci, S., Venturi, T., Bardelli, S., et al. 2006, NewA, 11, 437

Giacintucci, S., Venturi, T., Macario, G., et al. 2008, A\&A, 486, 347 
Grevesse, N., \& Sauval, A. J. 1998, SSRv, 85, 161

Hoeft, M., \& Brüggen, M. 2007, MNRAS, 375, 77

Hoeft, M., Brüggen, M., Yepes, G., Gottlöber, S., \& Schwope, A. 2008, MNRAS, 391, 1511

Kalberla, P. M. W., Burton, W. B., Hartmann, D., et al. 2005, A\&A, 440, 775

Kang, H., \& Jones, T. W. 2007, APh, 28, 232

Kang, H., \& Ryu, D. 2011 a, MmSAI, 82, 648

Kang, H., \& Ryu, D. 2011b, ApJ, 734, 18

Kuntz, K. D., \& Snowden, S. L. 2000, ApJ, 543, 195

Kuntz, K. D., \& Snowden, S. L. 2008, A\&A, 478, 575

Leccardi, A., \& Molendi, S. 2008, A\&A, 486, 359

Lumb, D. H., Warwick, R. S., Page, M., \& De Luca, A. 2002, A\&A, 389, 93

Macario, G., Markevitch, M., Giacintucci, S., et al. 2011, ApJ, 728, 82

Markevitch, M. 2010, arXiv:1010.3660

Markevitch, M., Gonzalez, A. H., David, L., et al. 2002, ApJL, 567, L27

Markevitch, M., Govoni, F., Brunetti, G., \& Jerius, D. 2005, ApJ, 627, 733
Mazzotta, P., Bourdin, H., Giacintucci, S., Markevitch, M., \& Venturi, T. 2011, MmSAI, 82, 495

Mazzotta, P., Rasia, E., Moscardini, L., \& Tormen, G. 2004, MNRAS, 354, 10

Pfrommer, C., Springel, V., Enßlin, T. A., \& Jubelgas, M. 2006, MNRAS, 367,113

Russell, H. R., Sanders, J. S., Fabian, A. C., et al. 2010, MNRAS, 406, 1721

Ryu, D., Kang, H., Hallman, E., \& Jones, T. W. 2003, ApJ, 593, 599

Smith, R. K., Brickhouse, N. S., Liedahl, D. A., \& Raymond, J. C. 2001, ApJL, 556, L91

Starck, J.-L., Fadili, J., \& Murtagh, F. 2007, ITIP, 16, 297

van Weeren, R. J., Brüggen, M., Röttgering, H. J. A., \& Hoeft, M. 2011, JApA, 32,505

Vazza, F., Brunetti, G., \& Gheller, C. 2009, MNRAS, 395, 1333

Venturi, T. 2011, MmSAI, 82, 499

Zhang, B., Fadili, J. M., \& Starck, J. L. 2008, IEEE Transactions on Image Processing, 17, 1093 